\title{
A Perspective on Immune Cell Metabolic Models for Host- based Therapeutic Discovery against COVID-19
}

\author{
Paulchamy Chellapandi*
}

Industrial Systems Biology Lab, Department of Bioinformatics, School of Life Sciences, Bharathidasan University, Tiruchirappalli, Tamil Nadu, India

\begin{abstract}
Severe acute respiratory syndrome coronavirus-2 (SARS-CoV-2) currently spread worldwide, causing the COVID-19 pandemic. The rate of its positive cases increases daily due to the unavailability of approved antivirals and a lack of knowledge of its pathophysiology in host-based antiviral discovery. Previous clinical data proved that SAS-CoV-2 infects human alveolar macrophages and naïve CD4 ${ }^{+} \mathrm{T}$ cells in lymphocytes in severe COVID-19 patients through cytokine storms. Like other viruses, SARS-CoV-2 infection reprograms cellular metabolism and deactivates innate immune systems and rely on metabolic changes in immune-specific cells for its optimal replication and pathogenesis. The interplay between cellular metabolism and innate immunity is thus of great interest in the development of host-based antivirals. Genome-scale metabolic networks of SARS-CoV-2-alveolar macrophage (iAB-AMØ-1410) and SARS-CoV-2-naïve CD4 ${ }^{+}$T cells (CD4T1670) have previously been reconstructed from the RECON1 model. These metabolic network models will be helpful for studying the interplay of cellular metabolism with immune responses in virus-infected and uninfected immune-specific cells. Such metabolic models could be used for a better understanding of its pathophysiology and host-based antiviral development.
\end{abstract}

Keywords: COVID-19; SARS-CoV-2; Immunometabolism; Genome-scale metabolic model; Antivirals; T-cells; Macrophages

\section{Introduction}

The novel coronavirus disease 2019 (COVID-19) is a global health concern caused by severe acute respiratory syndrome coronavirus-2 (SARSCoV-2). This virus replicates in the upper and lower respiratory tract, forming lesions. It shows symptoms slowly over an incubation period of around 2 weeks. The severe symptom of this virus progresses to severe pneumonia and acute respiratory distress syndrome. As of 10 February 2021 , the World Health Organization (WHO) has reported that 107M confirmed cases and 2.34M death cases have been confirmed worldwide [1]. Lifesupport is needed to treat severe COVID-19 patients. Several medications have been recommended in the treatment of mild to severe COVID-19 patients by the WHO and numerous drugs are also undergoing clinical trials [2]. Current efforts and treatment options hamper to overcome of its spread worldwide owing to a lack of knowledge of the host-pathogen biology of SARS-CoV-2. Hence, understanding its molecular pathogenesis and metabolic programming in host immune systems will offer important insights into the treatment and management of COVID-19.

\section{Pathobiology}

During viral infection, a host factor elicits an immune response against its invasion and infectivity. SARS-CoV-2 infects cells expressing ACE2 and TMPRSS2, replicates, and release viral particles in host cells to undergo pyroptosis and release damage-associated molecular patterns (ATP, nucleotides, etc.). Airway and alveolar epithelial cells, vascular endothelial cells, and macrophages recognize these factors and trigger the generation of pro-inflammatory cytokines and chemokines, and macrophage inflammatory proteins [3,4]. These proteins attract monocytes, macrophages, and T-cells to the site of infection to establish a pro-inflammatory feedback loop. Such defective immune response causes the overproduction of pro-inflammatory cytokines and then eventually damages the lung infrastructure. The resulting cytokine storm circulates to other organs, leading to multi-organ damage. Besides, SARS-CoV-2 infection triggers antibody-dependent enhancement by producing non-neutralizing antibodies and exacerbate further organ damage. In the meanwhile, inflammation reactions attract virus-specific T-cells to the site of infection for the elimination of infected cells before the virus spreads. Alveolar macrophages recognize neutralized viruses and apoptotic cells to clear virus loads through phagocytosis. These are the possible host immune responses against SARS-CoV-2 and minimal lung damage, resulting in recovery.

*Correspondence to: Paulchamy Chellapandi, Industrial Systems Biology Lab, Department of Bioinformatics, School of Life Sciences, Bharathidasan University, Tiruchirappalli-620024, Tamil Nadu, India; E-mail: pchellapandi@gmail.com

Citation: Chellapandi P (2021) A Perspective on Immune Cell Metabolic Models for Host-based Therapeutic Discovery against COVID-19. J Clin Anat Pathol, 6(1): 111. DOI: https://doi.org/10.47275/2332-4864-111

Received: November 26, 2020; Accepted: February 11, 2021; Published: February 18, 2021

Copyright: $\odot 2021$ Chellapandi P. This is an Open Access article distributed under the terms of the Creative Commons Attribution 4.0 International License (CCBY) (http://creativecommons.org/licenses/by/4.0/) which permits commercial use, including reproduction, adaptation, and distribution of the article provided the original author and source are credited. 


\section{Macrophage metabolism}

The viral replication process depends on the availability of biochemical building blocks and energy transducers in hosts. The mechanisms through which viruses modulate cellular metabolism to support viral infection are the important ones to identify potential therapeutic targets to block viral-associated diseases. Viral infectivity can be increased with activation of metabolism and inactivation of immune responses. It states the interplay between viral rewired metabolism and innate immunity (cytokine storm) in infected cells. The lower respiratory tract cells and upper cells in the pharyngeal region are targets for SARS-CoV-2 infectivity [5,6]. The SARS-CoV-2 infection causes pyroptosis in human alveolar macrophages and lymphocytes [3,4 and 7]. Severe SARS-CoV-2 infection induces peripheral blood lymphopenia in $82.1 \%$ of patients, suggestive of pulmonary infiltration of lymphocytes and/or cell damage through apoptosis or pyroptosis [6,8]. The pro-inflammatory and anti-inflammatory macrophages need fatty acids to produce cytokines and nitrous oxide in their polarized state. After activation, macrophages rely on glycolysis (Warburg effect) and the TCA cycle. Furthermore, virus-infected macrophages can shift their metabolism and synthesize anti-inflammatory lipid derivatives depending on host conditions. A mechanism behind cellular metabolism in host immune responses against SARS-CoV-2 infection remains unresolved. Hence, a metabolic model of human alveolar macrophages is selected as a host model to investigate crosstalk between cellular metabolism and innate immune systems to clearance of SARS-CoV-2.

\section{T-cell metabolism}

$\mathrm{CD}^{+} \mathrm{T}$-cells are necessary to nullify virus-infected cells, which are decisive to prime $\mathrm{CD} 8^{+} \mathrm{T}$-cells and $\mathrm{B}$ cells. These cells are also accountable for cytokine secretion to drive immune cell recruitment. Naïve T-cells activate, proliferate, and differentiate into different subtypes (Th1, Th2, Th17, and effector T cells) that mediate immune responses toward the invasion. Treg cells are mainly used to keep all the immune process of effector cells under control. Fatty acid oxidation and the TCA cycle are mainly used for energy production and other metabolic processes to keep immune surveillance in naïve T-cells. After activation, the metabolic machinery is changed to coordinate aerobic glycolysis, glutaminolysis, fatty acid oxidation, amino acids, and nucleotide metabolism. Cells turn to apoptosis and became memory T-cells for responding to future viral exposure. Hence, metabolic dysfunction could result in anergy in $\mathrm{CD}^{+} \mathrm{T}$-cells, but more detailed mechanisms are still unknown. Xu Z, et al. (2020) detected both T and B cell responses against SARS-CoV-2 in the blood around 1 week after the onset of COVID-19 symptoms [9]. The low levels of CD3 ${ }^{+}$, CD4 ${ }^{+}$, and CD8 ${ }^{+}$ T-lymphocytes were noticed in COVID-19 patients with lymphocytopenia [10]. SARS-CoV-2 infects T-lymphocytes through its spike proteinmediated membrane fusion [11]. A recent study has identified and characterized SARS-CoV-2-reactive T-cells in COVID-19 patients and healthy donors. The presence of them in a subset of SARS-CoV-2 naïve healthy donors is of high interest for the characterization of potential cross-reactive cellular immunity to SARS-CoV-2. Nevertheless, larger-scale prospective cohort studies are needed to assess whether their presence is a correlate of protection or pathology for COVID-19 [12]. Since SARS-CoV-2-reactive T-cells are important to eliminate viral loads and disease progression, a trial study on the metabolism of naïve T-cells and viral-infected cells will reach clinical purposes [13].

\section{Macrophage metabolic model}

Antiviral target discovery is of great interest, which can be recently achieved by analysis of metabolic changes in infected cells using constraintbased metabolic simulations. Antiviral targets for antiviral therapies can be validated by using reaction knockouts and host-derived enforcement approaches. The effect of metabolic reprogramming on host- and virus-optimized states can be studied by optimizing either for host maintenance or virus biomass objective function. Genome-scale metabolic models for specific immune cells such as a cell-specific alveolar macrophage model, iABAMØ-1410 [14], and a murine macrophage cell line, RAW $264.7[15,16]$ have been developed by integration of multi-omics datasets (Table 1). These models can be used to assess metabolic features that are critical for macrophage activation and to determine the metabolic immunomodulators of the cellular activation. Metabolic fluxes of nitrous oxide and ATP synthesis, and cell growth rate predicted with these models had better analogy than Recon 1 with in vitro results. Moreover, the human macrophage model, iAB-AMØ-1410 is integrated with the biochemical demands arising from virus production for the development of a genome-scale metabolic model of human-virus interaction. This model can predict the host-based antiviral targets against Chikungunya, Dengue, and Zika viruses and determine the impact of the virus on host metabolism and vice versa [17].

Comprehensive knowledge of host-virus interactions is central to devise host-based therapeutic strategies. Host-virus PPI networks provide key information to select targets for antiviral drug development. Herein, we have constructed virus-host protein interaction networks for Chikungunya virus (strain S27-African prototype) and Dengue virus type 2 (strain Jamaica/1409/1983) based on available experimental data (Figure 1). It describes that many virus proteins are associated with host proteins that are directly involved in innate immune systems including interleukin-1 receptor-associated kinase 3, interferon beta 1, and GTPase activating protein. RNA-directed RNA polymerase of Chikungunyavirus is associated with Ras homolog enriched in brain, microtubule-associated protein tau, and neurofilament, a heavy polypeptide of human. It reflects that this virus can affect human brain cells by interacting with structural proteins. The capsid protein of this virus interacts with human poly (ADP-ribose) polymerase family members and mind bomb E3 ubiquitin-protein ligase 1. It suggests that cellular processes such as DNA repair, genomic stability, and apoptosis mediated by poly (ADP-ribose) polymerase can be influenced with capsid protein of this virus in Chikungunya patients. Envelop protein E of Dengue virus type 2 can interact with polypyrimidine tract binding proteins and actin, suggesting that it can destabilize the cytoskeleton

Table 1: Overview of basic features of genome-scale metabolic models of macrophage and naïve CD4 ${ }^{+}$T-cells.

\begin{tabular}{|c|c|c|}
\hline Model properties & Alveolar macrophage cell & Nä̈ve CD4 T-cell \\
\hline Model name & iAB-AMØ-1410 & 1410 \\
\hline Genes & 3784 & 1670 \\
\hline Reactions & $69.30 \%$ & 7829 \\
\hline Gene associated reactions & 2583 & $78.30 \%$ \\
\hline
\end{tabular}




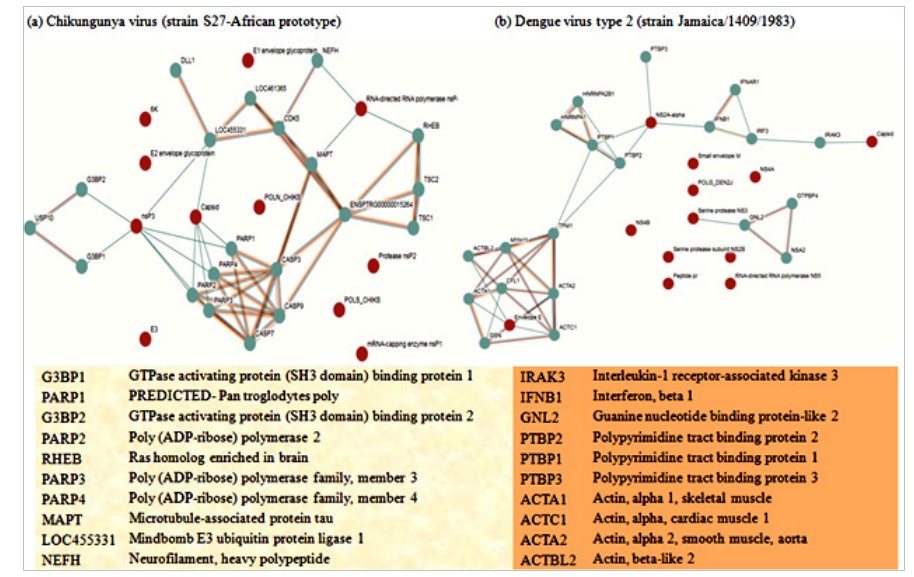

Figure 1: Virus-host protein interaction networks constructed from available experimental data using STRING Viruses database [18].

network of human cells. The capsid protein of this virus is associated with interleukin-1 receptor-associated kinase 3, which forms a protein network with Interferon beta 1. It represents the molecular involvement of capsid protein in human immunometabolism and innate immune response against virus spreading. Hence, host factors of SARS-CoV-2 replication are often found in key topological positions within PPI networks.

\section{T-cell metabolic model}

Recently, a genome-scale metabolic model of naïve CD4+T-cells (CD4T1670) has been reconstructed by integrating omics datasets (Table 1). It can be used to study the role of central metabolism in host immune responses and evaluate the side-effects of certain drugs in preclinical experiments [19]. Several omics studies have been characterized the metabolic phenotypes of CD4+ T-cells and their subsets (Th1, Th2, Th17), which will provide an opportunity to reconstruct T-helper specific metabolic models at the genome-scale [20-22]. Therefore, previous studies on metabolism and immune responses of macrophages and T-cells against viral infections, currently available omics data, and metabolic models will allow us for a better understanding of pathophysiology towards the development of host-based antivirals.

\section{Conclusion}

Viral infection frequently perturbs several cellular processes in the infected host and then causes severe diseases. During viral infection, host proteins can form a new complex with viral proteins through protein-protein interaction networks. The availability of large-scale virus-host interaction data will boost our knowledge of immunometabolism in virus-infected cells. This will lead to a more detailed understanding of viral pathogenesis and provide potential new targets for interfering with either the virus or host at key points in the infection.

The present preliminary study on virus-host protein interaction networks suggests the molecular involvement of some viral proteins in immunerelated metabolic pathways, host immune responses, and signal transduction process in humans. The interplay between cellular metabolism and the innate immune system is thus of great interest in the development of host-based antivirals. In this perspective, the genome-scale metabolic networks of SARS-CoV-2-alveolar macrophages and SARS-CoV-2-naïve CD4 ${ }^{+}$T-cells should be reconstructed for studying the interplay of cellular metabolism with immune responses in virus-infected and uninfected immune-specific cells. Such metabolic models will be more suitable for the investigation of drug action mechanisms and drug ability evaluation during SARS-CoV-2 infection. The systems biology platform developed from this perspective will allow us to understand its pathophysiology and pathogenesis mechanism to develop the host-based antivirals.

\section{Acknowledgement}

The author would like to thank the Science and Engineering Research Board, Department of Science and Technology (MSC/2020/000438), New Delhi, India, for financial support.

\section{Conflict of interest}

I have no conflict of interest.

\section{References}

1. Worldometer (2020) COVID-19 Coronavirus Pandemic.

2. Chellapandi P, Saranya S (2020) Genomics insights of SARS-CoV-2 (COVID-19) into target-based drug discovery. Medicinal Chemy Res 20: 1-15. https://doi.org/10.1007/s00044020-02610-8

3. CD4 ${ }^{+}$Y, Zhao Z, Wang Y, Zhou Y, Ma Y, et al. (2020) Single-cell RNA expression profiling of ACE2, the putative receptor of Wuhan 2019-nCoV. Am J Respir Crit Care Med 202: 756-759. https://doi.org/10.1101/2020.01.26.919985

4. Zhou P, Yang XL, Wang XG, Hu B, Zhang L, et al. (2020) A pneumonia outbreak associated with a new coronavirus of probable bat origin. Nature 579: 270-273. https://doi. org/10.1038/s41586-020-2012-7

5. Chen Y, Liu Q, Guo D (2020) Emerging coronaviruses: Genome structure, replication, and pathogenesis. J Med Virol 92: 418-423. https://doi.org/10.1002/jmv.25681 
Citation: Chellapandi P (2021) A Perspective on Immune Cell Metabolic Models for Host-based Therapeutic Discovery against COVID-19. J Clin Anat Pathol, 6(1): 111. DOI: https://doi.org/10.47275/2332-4864-111

6. Huang C, Wang Y, Li X, Ren L, Zhao J, et al. (2020) Clinical features of patients infected with 2019 novel coronavirus in Wuhan, China. Lancet 395: 497-506. https://doi.org/10.1016/ S0140-6736(20)30183-5

7. Yang P, Wang X (2020) COVID-19: a new challenge for human beings. Cell Mol 17: 555-557. https://doi.org/10.1038/s41423-020-0407-x

8. Guan w, Ni z, Hu Y, Liang w, Ou C, et al. (2020) Clinical characteristics of coronavirus disease 2019 in China. N Engl J Med 382: 1708-1720. https://doi.org/10.1056/NEJMoa2002032

9. Xu Z, Shi L, Wang Y, Zhang J, Huang L, et al. (2020) Pathological findings of COVID-19 associated with acute respiratory distress syndrome. Lancet Respir Med 8:420-422. https:// doi.org/10.1016/S2213-2600(20)30076-X

10. Zheng M, Gao Y, Wang G, Song G, Liu S, et al. (2020) Functional exhaustion of antiviral lymphocytes in COVID-19 patients. Cell Mol Immunol 17: 533-535. https://oi.org/10.1038/ s41423-020-0402-2

11. Wang X, Xu W, Hu G, Xia S, Sun Z, et al. (2020) SARS-CoV-2 infects T lymphocytes through its spike protein-mediated membrane fusion. Cell Mol Immunol 18: 1-3. https://doi. org/10.1038/s41423-020-0424-9

12. Braun J, Loyal L, Frentsch M, Wendisch D, Georg P, et al. (2020) Presence of SARS-CoV-2-reactive T cells in COVID-19 patients and healthy donors. Nature 587: 270-274. https:// doi.org/10.1101/2020.04.17.20061440

13. de Candia P, Prattichizzo F, Garavelli S, Matarese G (2021) T Cells: Warriors of SARS-CoV-2 Infection. Trends Immunol 42: 18-30. https://doi.org/10.1016/j.it.2020.11.002

14. Bordbar A, Lewis NE, Schellenberger J, Palsson BØ, Jamshidi N (2010) Insight into human alveolar macrophage and M. tuberculosis interactions via metabolic reconstructions. Mol Syst Biol 6: 422. https://doi.org/10.1038/msb.2010.68

15. Duarte NC, Becker SA, Jamshidi N, Thiele I, Mo ML, et al. (2007) Global reconstruction of the human metabolic network based on genomic and bibliomic data. Proc Natl Acad Sci USA 104: 1777-1782. https://doi.org/10.1073/pnas.0610772104

16. Bordbar A, Mo ML, Nakayasu ES, Schrimpe-Rutledge AC, Kim YM, et al. (2012) Model-driven multi-omic data analysis elucidates metabolic immunomodulators of macrophage activation. Mol Syst Biol 8: 558. https://doi.org/10.1038/msb.2012.21

17. Aller S, Scott A, Sarkar-Tyson M, Soyer OS (2018) Integrated human-virus metabolic stoichiometric modeling predicts host-based antiviral targets against Chikungunya, Dengue, and Zika viruses. J R Soc Interface 15: 20180125. https://doi.org/10.1098/rsif.2018.0125

18. Cook HV, Doncheva NT, Szklarczyk D, von Mering C, Jensen LJ (2018) Viruses.STRING: A Virus-Host Protein-Protein Interaction Database. Viruses 10:519. https://doi.org/10.3390/ v10100519

19. Han F, Li G, Dai S, Huang J (2016) Genome-wide metabolic model to improve understanding of CD4(+) T cell metabolism, immunometabolism and application in drug design. Mol Biosyst 12:431-443. https://doi.org/10.1039/c5mb00480b

20. Pollizzi KN, Powell JD (2014) Integrating canonical and metabolic signalling programmes in the regulation of T cell responses. Nat Rev Immunol 14:435-446. https://doi.org/10.1038/ nri3701

21. Kanduri K, Tripathi S, Larjo A, Mannerstrom H, Ullah U, et al. (2015) Identification of global regulators of T-helper cell lineage specification. Genome Med 7:122. https://doi. org/10.1186/s13073-015-0237-0

22. Tuomela S, Rautio S, Ahlfors H, Oling V, Salo V, et al. (2016) Comparative analysis of human and mouse transcriptomes of Th17 cell priming. Oncotarget 7: 13416-13428. https:// doi.org/10.18632/oncotarget. 7963 